\title{
Locating Defects in Conductive Materials Using the Eddy Current Model of the Filamentary Coil
}

\author{
Grzegorz Tytko ${ }^{1}$ (D)
}

Received: 15 February 2021 / Accepted: 12 July 2021 / Published online: 31 July 2021

(C) The Author(s) 2021

\begin{abstract}
This article presents an innovative technique for detecting flaws in conductive materials by using an ideal filamentary coil. To characterize such a coil accurately and explicitly, it is sufficient to be in possession of merely two parameters: the radius of the circle within which all the turns are located and the distance of the coil from the tested surface. The mathematical model derived using the truncated region eigenfunction expansion method enables the calculation of the changes in the components of the filamentary coil impedance that are the result of positioning the coil close to the conductive material with a hole. Because of this, the air-cored coil model can be replaced with a much simpler filamentary coil model. This solution makes it is possible to detect various types of holes (internal, surface, subsurface or through) occurring in both multilayer magnetic and non-magnetic materials. The derived results were verified by means of measurements and numerical calculations based on the finite element method. Very good agreement was observed in both cases. The paper contains the source code implemented in Matlab, which is used to for calculations.
\end{abstract}

Keywords Eddy current testing $\cdot$ Filamentary coil $\cdot$ Truncated region eigenfunction expansion $\cdot$ Locating defects $\cdot$ Coil impedance

\section{Introduction}

One of the most frequently used methods of interpreting the results obtained in eddy current testing uses theoretical analysis to predict the changes of the probe impedance components [1-5]. The probe may be an alternating current fed coil, the impedance of which changes because of the probe fields interaction defects in the tested material. The value of these impedance changes depends on many parameters relating to the defect itself (geometric dimensions, location), the probe used (shape, excitation frequency, number of turns) and the tested material (electrical conductivity, magnetic permeability). The employment of an air-cored coil as a probe for detecting flaws in conductors was analysed for magnetic [6-9] and non-magnetic [10-14] materials. What is commonly used in the process of interpreting the changes in the coil impedance are mathematical models which make

Grzegorz Tytko

grzegorz.tytko@polsl.pl

1 Faculty of Automatic Control, Electronics and Computer Science, The Silesian University of Technology, Akademicka 16, Gliwice, Poland it possible to carry out simulations of the tests being done. In such models, the geometry of the air-cored coil is determined by its height $h_{2}-h_{1}$ and width $r_{2}-r_{1}$ (Fig. 1). However, in many cases, e.g. while designing a defectoscope, constructing the scale of a measuring instrument or determining optimal parameters of the test, this solution is insufficient. The employment of the four parameters determining the geometric dimensions of the coil turns out to be inconvenient and time-consuming. The most advantageous approach to use for dealing with this type of problem is to adopt the simplest known mathematical model, which is to use an equivalent ideal filamentary coil as a model of a multi-turn coil. In such a coil, located at a distance $h_{0}$ from the surface of the tested material, all turns are concentrated in one circle of radius $r_{0}$ (Fig. 2). Thus, only two parameters are sufficient to explicitly describe a filamentary coil, i.e. radius $r_{0}$ and distance $h_{0}$.

The technique of replacing the mathematical model of an air-cored coil with an ideal filamentary coil was developed for the needs of eddy current conductometry $[15,16]$. Following this method, each cylindrical air-cored coil can be assigned such a particular pair of parameters $r_{0}, h_{0}$, that the change in the impedance of both coils is the same. The novelty of this 


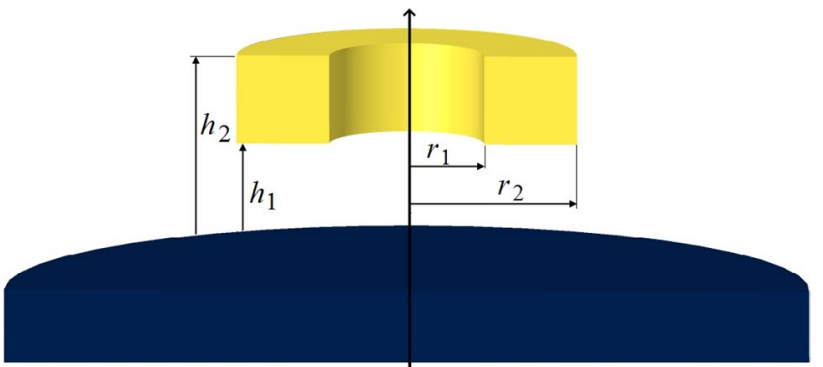

Fig. 1 Air-cored coil above a conductive material

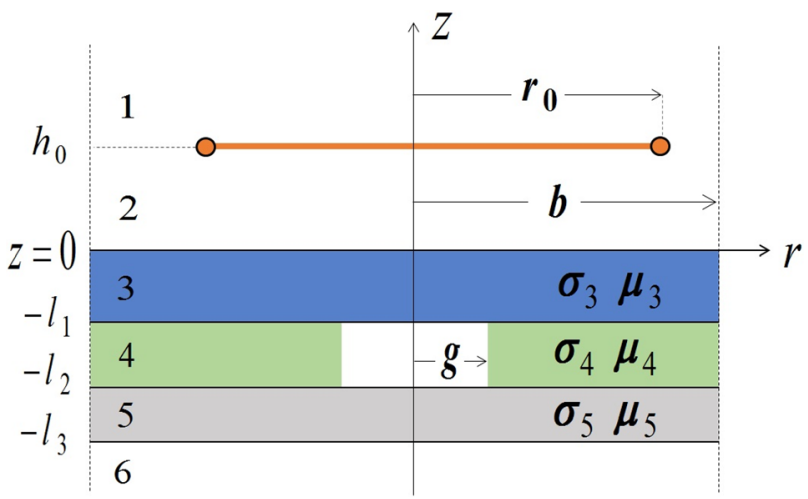

Fig. 2 Rectangular cross-sectional filamentary coil located above a three-layer conductive plate with an inner hole

paper consists in the adaptation of this property for detecting flaws in both magnetic and non-magnetic materials. For this purpose, an analytical model of a filamentary coil placed above a three-layer plate with a hole was developed. The final formulas were correlated to the mathematical model of an air-cored coil to obtain an analytical expression enabling efficient determination of parameters $r_{0}, h_{0}$. These values are calculated only once, in such a way that each change of the parameters, such as the excitation frequency of the system under consideration causes the same change in the impedance of the air-cored and filamentary coil. As a result, the mathematical model of an air-cored coil can be replaced with a much simpler model of a filamentary coil while maintaining the same accuracy of calculations. The presented solution allows modelling the presence of various types, such as surface, subsurface, through or internal holes of any size. The correctness of the obtained results was confirmed by the experiment and the finite element method (FEM).

\section{Methods}

The cross-section of the filamentary coil in a cylindrical coordinate system is shown in Fig. 2. All turns of the coil were placed in a circle of radius $r_{0}$ at a distance $h_{0}$ from the surface of a three-layer plate of electrical conductivities $\sigma_{3}, \sigma_{4}, \sigma_{5}$ and magnetic permeabilities $\mu_{3}, \mu_{4}, \mu_{5}$. A hole of radius $g$ and depth $l_{2}-l_{1}$ was made in the central layer of the plate. Subsequently, a mathematical model was developed using the truncated region eigenfunction expansion (TREE) method [17]. The analysis is based on the expressions for a filamentary coil positioned over a conductive half-space [18] and an I-core coil over a three-layer plate with a hole [19], the magnetic vector potential for each region was formulated as follows:

$\mathrm{A}_{1}(r, z)=J_{1}(\mathbf{q} r) e^{-\mathbf{q} z} \mathbf{C}_{1}$,

$\mathrm{A}_{2}(r, z)=J_{1}(\mathbf{q} r)\left(e^{-\mathbf{q} z} \mathbf{C}_{2}-e^{\mathbf{q} z} \mathbf{B}_{2}\right)$,

$\mathrm{A}_{3}(r, z)=J_{1}(\mathbf{q} r)\left(e^{-\mathbf{s}_{3} z} \mathbf{C}_{3}-e^{\mathbf{s}_{3} z} \mathbf{B}_{3}\right)$,

$\mathrm{A}_{4}(r, z)=\begin{array}{ll}J_{1}(\mathbf{u} r) P_{1}(\mathbf{v} g) \\ P_{1}(\mathbf{v} r) J_{1}(\mathbf{u} g)\end{array}\left(e^{-\mathbf{u} z} \mathbf{C}_{4}-e^{\mathbf{u} z} \mathbf{B}_{4}\right), \begin{aligned} & 0 \leq r \leq g \\ & g \leq r \leq b\end{aligned}$

$\mathrm{A}_{5}(r, z)=J_{1}(\mathbf{q} r)\left(e^{-\mathbf{s}_{5} z} \mathbf{C}_{5}-e^{\mathbf{s}_{5} z} \mathbf{B}_{5}\right)$,

$\mathrm{A}_{6}(r, z)=-J_{1}(\mathbf{q} r) e^{\mathbf{q} z} \mathbf{B}_{6}$,

where $J_{1}(\mathbf{q} r), J_{1}(\mathbf{u} r), J_{1}(\mathbf{u} g), P_{1}(\mathbf{v} g), P_{1}(\mathbf{v} r)$ are row vectors, $\mathbf{q}, \mathbf{u}, \mathbf{v}, \mathbf{e}^{\mathbf{x}}$ are diagonal matrices, $\mathbf{C}_{\mathrm{i}}, \mathbf{B}_{\mathrm{i}}$ are column vectors of unknown coefficients, whereas $P_{\mathrm{n}}(\mathbf{v} x)$ has been defined in terms of a Bessel function of the second kind; $Y_{1}(x)$, as:

$P_{\mathrm{n}}(\mathbf{v} x)=Y_{1}(\mathbf{v} b) J_{\mathrm{n}}(\mathbf{v} x)-J_{1}(\mathbf{v} b) Y_{\mathrm{n}}(\mathbf{v} x)$.

The eigenvalues $\mathbf{q}$ are successive roots of function $J_{1}(x)$ divided by the limitation of the solution domain $b$, hence $\mathbf{q}=\mathbf{x} / b$, whereas the material coefficients $\mathbf{s}_{\mathrm{n}}$ were calculated using the equation $\mathbf{s}_{\mathrm{n}}=\left(\mathbf{q}^{2}+j \omega \mu_{\mathrm{n}} \mu_{0} \sigma_{\mathrm{n}}\right)^{1 / 2}$. Region 4 was divided into 2 subregions containing respectively: a hole $(0 \leq r \leq g)$ and conductive material $(g \leq r \leq b)$. Complex eigenvalues $\mathbf{u}$ of this region and the corresponding coefficients $\mathbf{v}=\left(\mathbf{u}^{2}-j \omega \mu_{4} \mu_{0} \sigma_{4}\right)^{1 / 2}$ were calculated with the expression:

$\mathbf{u} P_{1}(\mathbf{v} g) J_{0}(\mathbf{u} g)=\frac{\mathbf{v}}{\mu_{4}} J_{1}(\mathbf{u} g) P_{0}(\mathbf{v} g)$.

Equation (8) can be solved utilising any method of finding complex roots of a complex function [20-23].

By using the expressions for the magnetic vector potential (1)-(6), the conditions for the continuity of the magnetic field (9)-(10) between adjacent regions were satisfied.

$A_{i}(r, z)=A_{i+1}(r, z) \quad i=1,2, \ldots, 5$. 
$\frac{1}{\mu_{i}} \frac{\partial A_{i}}{\partial z}-\frac{1}{\mu_{i+1}} \frac{\partial A_{i+1}}{\partial z}=-\mu_{0} I \delta\left(r-r_{0}\right) \quad i=1,2, \ldots, 5$.

The current density in the filament is $\mu_{0} I \delta\left(r-r_{0}\right)$. Solving the above system of 10 interface equations results in the determination of the unknown coefficients $\mathbf{C}_{\mathrm{i}}, \mathbf{B}_{\mathrm{i}}$, which are normalised as follows: $\mathbf{C}_{\mathrm{i} 6}=\mathbf{C}_{\mathrm{i}} / \mathbf{B}_{6}, \mathbf{B}_{\mathrm{i} 6}=\mathbf{B}_{\mathrm{i}} / \mathbf{B}_{6}$.

$$
\begin{aligned}
& \mathbf{B}_{26}=-\left[\left(\frac{1}{\mu_{3}}-\mathbf{q} \mathbf{s}_{3}^{-1}\right) \mathbf{C}_{36}+\left(\frac{1}{\mu_{3}}+\mathbf{q} \mathbf{s}_{3}^{-1}\right) \mathbf{B}_{36}\right] \\
& \mathbf{C}_{26}=\left[\left(\frac{1}{\mu_{3}}+\mathbf{q} \mathbf{s}_{3}^{-1}\right) \mathbf{C}_{36}+\left(\frac{1}{\mu_{3}}-\mathbf{q} \mathbf{s}_{3}^{-1}\right) \mathbf{B}_{36}\right] \\
& \mathbf{B}_{36}=e^{\mathbf{s}_{3} l_{1}}\left[\left(\mu_{3} \mathbf{V}-\mathbf{s}_{3} \mathbf{u}^{-1} \mathbf{K}\right) e^{\mathbf{u} l_{1}} \mathbf{C}_{46}\right. \\
& \left.+\left(\mu_{3} \mathbf{V}+\mathbf{s}_{3} \mathbf{u}^{-1} \mathbf{K}\right) e^{-\mathbf{u} l_{1}} \mathbf{B}_{46}\right], \\
& \mathbf{C}_{36}=e^{-\mathbf{s}_{3} l_{1}}\left[\left(\mu_{3} \mathbf{V}+\mathbf{s}_{3} \mathbf{u}^{-1} \mathbf{K}\right) e^{\mathbf{u} l_{1}} \mathbf{C}_{46}\right. \\
& \left.+\left(\mu_{3} \mathbf{V}-\mathbf{s}_{3} \mathbf{u}^{-1} \mathbf{K}\right) e^{-\mathbf{u} l_{1}} \mathbf{B}_{46}\right] \text {, } \\
& \mathbf{B}_{46}=e^{\mathbf{u} l_{2}}\left[\left(\frac{\mathbf{V}^{-1}}{\mu_{5}}-\mathbf{s}_{5}^{-1} \mathbf{u} \mathbf{K}^{-1}\right) e^{\mathbf{s}_{5} l_{2}} \mathbf{C}_{56}+\left(\frac{\mathbf{V}^{-1}}{\mu_{5}}+\mathbf{s}_{5}^{-1} \mathbf{u} \mathbf{K}^{-1}\right) e^{-\mathbf{s}_{5} l_{2}} \mathbf{B}_{56}\right], \\
& \mathbf{C}_{46}=e^{-\mathbf{u} l_{2}}\left[\left(\frac{\mathbf{V}^{-1}}{\mu_{5}}+\mathbf{s}_{5}^{-1} \mathbf{u} \mathbf{K}^{-1}\right) e^{\mathbf{s}_{5} l_{2}} \mathbf{C}_{56}+\left(\frac{\mathbf{V}^{-1}}{\mu_{5}}-\mathbf{s}_{5}^{-1} \mathbf{u} \mathbf{K}^{-1}\right) e^{-\mathbf{s}_{5} l_{2}} \mathbf{B}_{56}\right],
\end{aligned}
$$

$\mathbf{C}_{56}=e^{-\mathbf{s}_{5} l_{3}}\left(\mu_{5}-\mathbf{s}_{5} \mathbf{q}^{-1}\right)$,

$\mathbf{V}=\left[v_{\mathrm{ij}}\right]=\boldsymbol{\beta}_{1}+\frac{1}{\mu_{4}} \boldsymbol{\beta}_{2}$,

$\boldsymbol{\beta}_{1}=\frac{g}{u_{\mathrm{j}}^{2}-q_{\mathrm{i}}^{2}} R_{1}\left(v_{\mathrm{j}} g\right)\left[q_{\mathrm{i}} J_{0}\left(q_{\mathrm{i}} g\right) J_{1}\left(u_{\mathrm{j}} g\right)-u_{\mathrm{j}} J_{1}\left(q_{\mathrm{i}} g\right) J_{0}\left(u_{\mathrm{j}} g\right)\right]$,

$\boldsymbol{\beta}_{2}=-\frac{g}{v_{\mathrm{j}}^{2}-q_{\mathrm{i}}^{2}} J_{1}\left(u_{\mathrm{j}} g\right)\left[q_{\mathrm{i}} J_{0}\left(q_{\mathrm{i}} g\right) R_{1}\left(v_{\mathrm{j}} g\right)-v_{\mathrm{j}} J_{1}\left(q_{\mathrm{i}} g\right) R_{0}\left(v_{\mathrm{j}} g\right)\right]$.

Expressions (11)-(18) were employed to develop the final formula for a change in the impedance of the filamentary coil, where the change resulted from placing the coil over a three-layer plate with an internal hole.
$\Delta Z=j 2 \pi \omega \mu_{0} r_{0}^{2} b N^{2} \mathbf{q}^{-1} J_{1}^{2}\left(\mathbf{q} r_{0}\right) J_{0}^{-2}(\mathbf{q} b) e^{-2 \mathbf{q} h_{0}} \frac{\mathbf{C}_{26}}{\mathbf{B}_{26}}$

The selection of the type of the analysed hole in the tested object is made by introducing appropriate values of the material parameters, e.g. electrical conductivity. In this way, it is possible to model:

- internal hole $\left(\sigma_{3}>0\right.$ and $\left.\sigma_{5}>0\right)$,

- surface hole $\left(\sigma_{3}=0\right)$,

- subsurface hole $\left(\sigma_{5}=0\right)$,

- through hole $\left(\sigma_{3}=0\right.$ and $\left.\sigma_{5}=0\right)$.

The replacement of the air-cored coil model with a filamentary coil requires the values of parameters $r_{0}, h_{0}$ to be determined in such a way that changes of the impedance of both coils are the same. For this purpose, two solutions may be adopted: one of them is to utilise the obtained in the measurement value of the change in the impedance of the air-cored coil. Another one is to make the calculations using a mathematical model. The solution for an air-cored coil placed over the conductive half-space [24] was extended

by the presence of a three-layer plate with an internal hole. The change in the impedance of such a coil can be calculated from:

$\Delta Z_{a}=\frac{j \omega 2 \pi \mu_{0} N^{2}}{\left(r_{2}-r_{1}\right)^{2}\left(h_{2}-h_{1}\right)^{2}} \frac{\chi^{2}\left(\mathbf{q} r_{1}, \mathbf{q} r_{2}\right)}{b^{2} \mathbf{q}^{7} J_{0}^{2}(\mathbf{q} b)}\left(e^{-\mathbf{q} h_{1}}-e^{-\mathbf{q} h_{2}}\right) \frac{\mathbf{C}_{26}}{\mathbf{B}_{26}}$,

where

$\chi^{2}\left(\mathbf{q} r_{1}, \mathbf{q} r_{2}\right)=\mathbf{q}^{2} \int_{r_{1}}^{r_{2}} r J_{1}(\mathbf{q} r) d r$

Having acquired the value of the change of the coil impedance $\Delta Z_{\mathrm{a}}$, values $r_{0}, h_{0}$, it is possible to calculate iteratively [25].

\section{Results}

The measurements of the air-cored coil impedance (Fig. 3) were carried out with an Agilent 4294A precision impedance analyser for the frequency range from 1 to $50 \mathrm{kHz}$. The 


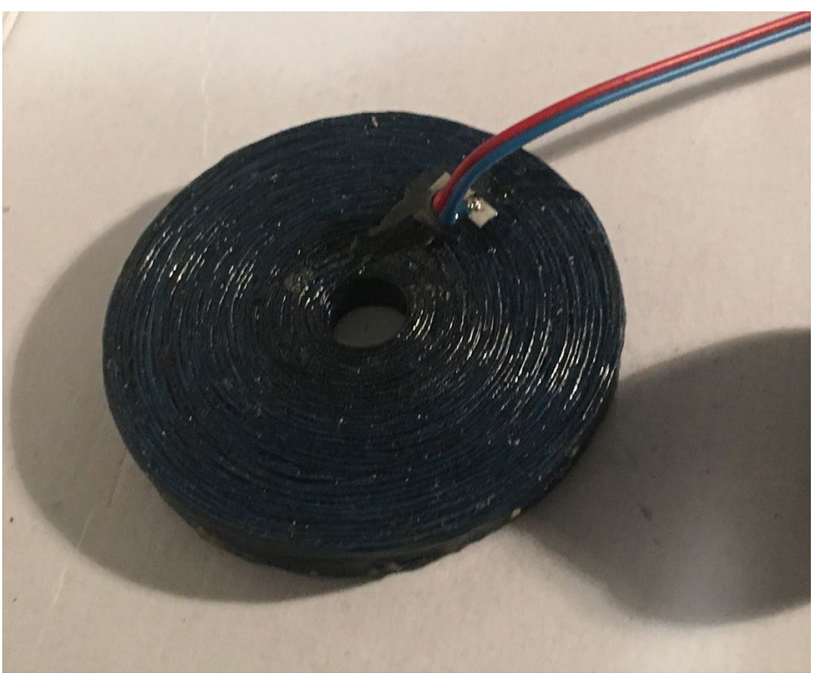

Fig. 3 Air-cored coil used for the measurements

Table 1 Parameters of the coil and sample used in measurements

\begin{tabular}{lll}
\hline Number of turns & $N$ & 700 \\
Inner coil radius & $r_{1}$ & $4.075 \mathrm{~mm}$ \\
Outer coil radius & $r_{2}$ & $25.85 \mathrm{~mm}$ \\
Coil length & $h_{2}-h_{1}$ & $6.2 \mathrm{~mm}$ \\
Radius of the hole & $g$ & $4 \mathrm{~mm}$ \\
Parameter & $l_{1}$ & $0.16 \mathrm{~mm}$ \\
Parameter & $l_{2}$ & $4.16 \mathrm{~mm}$ \\
Parameter & $l_{3}$ & $4.32 \mathrm{~mm}$ \\
Conductivity & $\sigma_{3}$ & $3.55 \mathrm{MSm}^{-1}$ \\
Conductivity & $\sigma_{4}$ & $13.45 \mathrm{MSm}^{-1}$ \\
Conductivity & $\sigma_{5}$ & $3.3 \mathrm{MSm}^{-1}$ \\
Relative permeability & $\mu_{3}, \mu_{4}, \mu_{5}$ & 1 \\
Radius of the domain & $b$ & $12 r_{2}$ \\
Summation terms & $N s$ & 100 \\
\hline
\end{tabular}

parameters of the used coil and the three-layer sample with an internal hole are presented in Table 1 . The value of the changes in the components of the coil impedance measured for the frequency $f=20 \mathrm{kHz}$ was used to determine parameters $r_{0}, h_{0}$ with the employment of the algorithm presented in [25]. Thus, measured values $r_{0}=17.3 \mathrm{~mm}, h_{0}=6.1 \mathrm{~mm}$ were used to calculate changes in the components of the filamentary coil impedance from (23), which were normalised with respect to the reactance of the coil in the free space $X_{0}$ and shown in Figs. 4 and 5. Subsequently, expression (24) was implemented in Matlab to compare the results obtained for the filamentary and air-cored coils. In both cases, in order to calculate the discrete eigenvalues in region 4 , the multilevel computation of complex eigenvalues (MCCE) method was used [23]. Additionally, the results were also verified using the finite element method. The numerical model of the air-cored coil created in the Comsol Multiphysics package

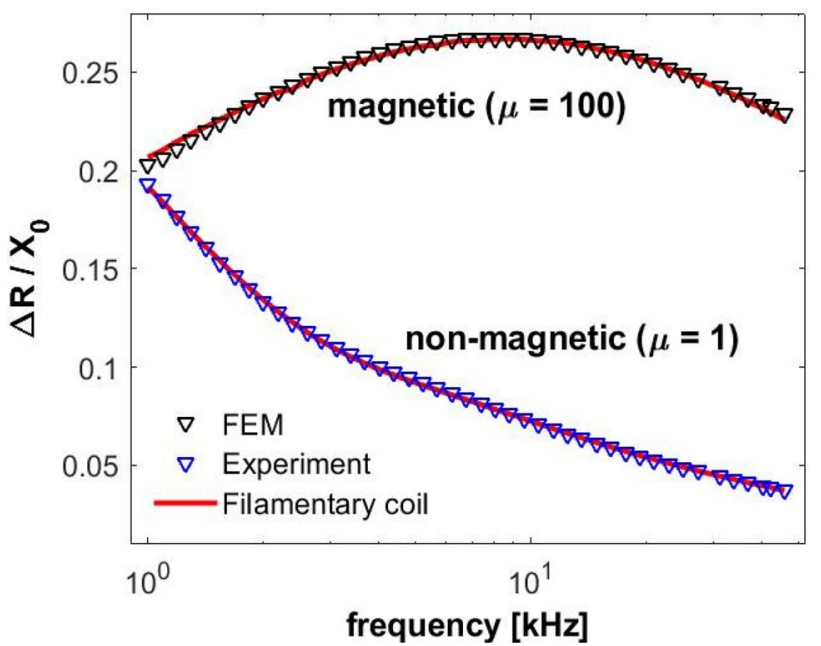

Fig. 4 Normalised changes in the coil resistance for magnetic material with a through hole and for non-magnetic material with an internal hole

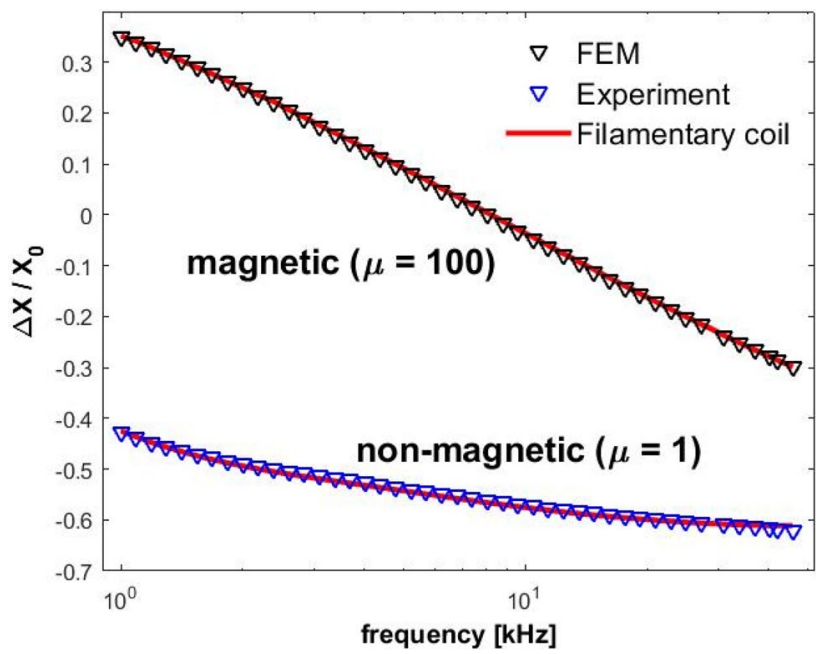

Fig. 5 Normalised changes in the coil reactance for magnetic material with a through hole and for non-magnetic material with an internal hole

consisted of 76,916 triangular elements. The obtained results of the changes of the coil impedance for several frequency values are presented in Table 2 .

In the case of the second solution, with the employment of the numerical model calculations were made for the same coil positioned at a distance of $0.1 \mathrm{~mm}$ from the surface of a $5 \mathrm{~mm}$ thick magnetic plate $(\mu=100)$ of electrical conductivity of $10.5 \mathrm{MSm}^{-1}$. A through hole of radius $g=4 \mathrm{~mm}$ was made in this plate. Afterwards, by using values $\Delta Z$ obtained for the frequency of $20 \mathrm{kHz}$, parameters $r_{0}=17.6 \mathrm{~mm}$, $h_{0}=6.1 \mathrm{~mm}$ were determined. These values were used to calculate the changes of the resistance and reactance of the filamentary coil (23). Results thus obtained are presented 
Table 2 Values of the changes in coil impedance $\Delta Z(\Omega)$ located above a three-layer plate with an inner hole

\begin{tabular}{llccc}
\hline$f(\mathrm{kHz})$ & Experiment & FEM & TREE air-cored coil (24) & TREE filamentary coil (23) \\
\hline 1 & $11.86-j 26.18$ & $11.99-j 25.68$ & $12.02-j 26.36$ & $11.75-j 26.04$ \\
20 & $66.37-j 735.77$ & $65.87-j 732.45$ & $65.86-j 745.87$ & $66.67-j 739.96$ \\
50 & $111.12-j 1946.78$ & $110.13-j 1891.70$ & $110.05-j 1925.10$ & $111.89-j 1911.79$ \\
\hline
\end{tabular}

in Figs. 4 and 5, whereas the values of the changes of the coil impedance components for selected frequencies are shown in Table 3. The source code implemented in Matlab, which enables carrying out the calculations, is included in the "Appendix".

\section{Discussion}

The maximum error of the changes in the impedance components calculated with the mathematical model of a filamentary coil (in comparison to the measured values) in the case of resistance and reactance was $1.5 \%$ and $1.8 \%$, respectively. These values are slightly lower than for the air-cored coil model (24), which were $2.2 \%$ and $2.4 \%$, respectively. The observed difference is the result of the adopted values (the same for both coils) of parameters $b$ and $N s$, on which the error of the TREE method is dependent. The first parameter is the radius of the solution domain. The increasing of $b$ results in a reduction of the calculation error, however it requires a larger value of $N s$. This parameter determines the number of the elements of vectors and matrices employed in the calculation. An increase of the value of $N s$ facilitates obtaining results with smaller error, however it requires carrying out more operations, which may significantly increase the time of receiving the calculation outcome. The author's observations show that a filamentary coil requires a lower value of $N s$ than an air-cored coil. Thus, calculations are performed faster not only due to the simpler form of expressions, but also because of a smaller number of mathematical operations that are necessary to perform [25]. In the case of the filamentary coil, $N s=100, b=12 r_{2}$, were adopted in the calculations, since a further increase of the values of these parameters did not bring about any significant improvement of the accuracy of the results.

Error control in the case of the filamentary coil is much easier than for the air-cored coil also due to the difference between parameters $h_{0}$ and $h_{1}$. The determination of the exact value of parameter $h_{1}$ may be troublesome, especially when the test sample is not polished. Also, the lower surface of the winding is not usually smooth. Due to these factors, a tiny air gap appears even when the coil comes into direct contact with the tested object. In computer modelling, its height $h_{1}$ is usually assumed to be about $0.05-0.2 \mathrm{~mm}$. This parameter has a great influence on the coil impedance, therefore even its slight deviation may significantly increase the calculation error. Nevertheless, this effect may be limited by using shields or washers under the coils while carrying out the tests. It is worth noting that this problem does not occur in the case of the filamentary coil, where the value of parameter $h_{0}$ is usually so large that minor changes in it do not have a significant influence on the results.

In the performed calculations, parameters $r_{0}, h_{0}$ were determined only once for the frequency $f=20 \mathrm{kHz}$. The selected frequency value was in the vicinity of the centre of the considered range. However, parameters $r_{0}, h_{0}$ can be determined as well, for example, for the lowest or the highest value of the parameter under study. This was not found to cause any significant increase or decrease in the calculation error. The time of determining parameters $r_{0}, h_{0}$ with a computer operated by an Intel Core i5 processor was only about $0.8 \mathrm{~s}$.

\section{Conclusions}

The developed method of detecting flaws in conductive materials makes it possible to successfully replace the mathematical model of an air-cored coil with an ideal filamentary coil. The values of $r_{0}, h_{0}$ are to be determined once since each change of the parameters of the system causes the same change in the impedance of both coils. The derived mathematical model enables performing effective calculations for various types of holes in both single- and multilayer plates. The calculations were made for an internal hole in a threelayer non-magnetic material $(\mu=1)$ and for a through hole
Table 3 Values of the changes in coil impedance $\Delta Z(\Omega)$ located above a magnetic plate with a through hole

\begin{tabular}{lllrr}
\hline$f(\mathrm{kHz})$ & FEM & TREE filamentary coil (23) & $\begin{array}{l}\text { Resistance error } \\
(\%)\end{array}$ & $\begin{array}{l}\text { Reac- } \\
\text { tance } \\
\text { error }(\%)\end{array}$ \\
\hline 1 & $12.61+j 21.66$ & $12.85+j 21.84$ & -1.85 & -0.82 \\
20 & $317.33-j 203.90$ & $317.45-j 201.26$ & -0.04 & 1.29 \\
50 & $700.86-j 966.40$ & $689.52-j 968.06$ & 1.62 & -0.17 \\
\hline
\end{tabular}


in a magnetic material $(\mu=100)$. The obtained results, in comparison with the measurements, were characterised by a small error not exceeding $2 \%$ for changes in both resistance and reactance.

The employment of the filamentary coil has many advantages: it reduces the time-cost of making calculations, obtaining a simpler form of analysis to improve the process of designing and calibrating of measuring instruments. In the mathematical model of the filamentary coil derived by the TREE method, the final formulas were written in a closed form. This enables writing the source code in any programming language ("Appendix") and using it with a measuring device equipped with a microprocessor. It is planned that in the future, further research will be carried out regarding the implementation of the presented solution in the defectoscope, and the extension of the mathematical model for systems containing several coils.

\section{Appendix}

The changes in the components of the filamentary coil impedance obtained for the magnetic material with a through hole (Table 3) can be obtained with the employment of this algorithm implemented in Matlab. The procedure zerobess $J 1(x)$ is used to find successive roots of the Bessel function $J_{1}(x)$. The eigenvalues in region 4 were calculated using the MCCE algorithm [23]. Instead of these, any method for finding complex roots of a complex function can be used as well.

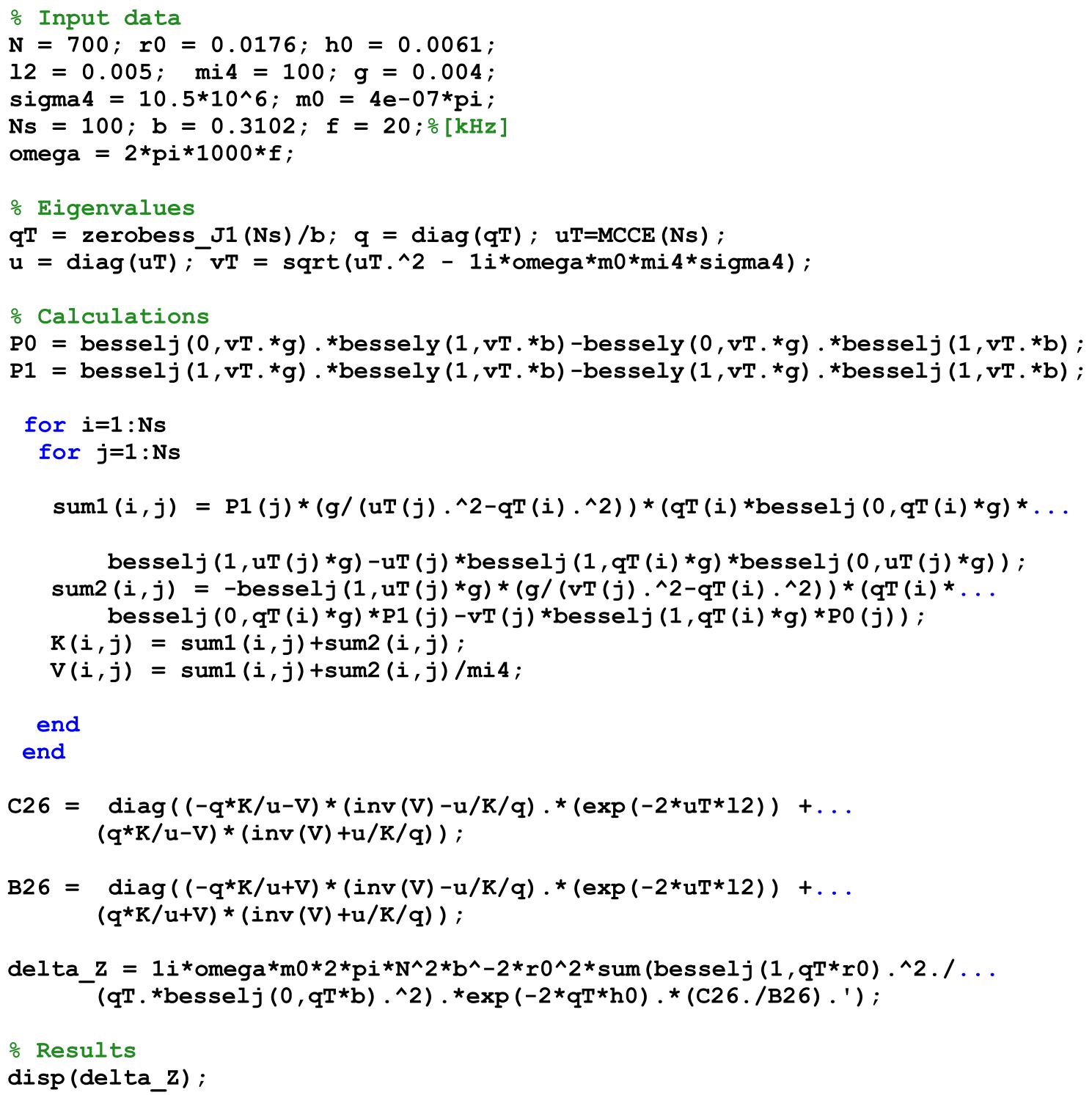




\section{Declarations}

Conflict of interest The author has no relevant financial or non-financial interests to disclose.

Open Access This article is licensed under a Creative Commons Attribution 4.0 International License, which permits use, sharing, adaptation, distribution and reproduction in any medium or format, as long as you give appropriate credit to the original author(s) and the source, provide a link to the Creative Commons licence, and indicate if changes were made. The images or other third party material in this article are included in the article's Creative Commons licence, unless indicated otherwise in a credit line to the material. If material is not included in the article's Creative Commons licence and your intended use is not permitted by statutory regulation or exceeds the permitted use, you will need to obtain permission directly from the copyright holder. To view a copy of this licence, visit http://creativecommons.org/licenses/by/4.0/.

\section{References}

1. Zhu, Y., Chen, B., Luo, Y., et al.: Inductance calculations for coils with an iron core of arbitrary axial position. Electromagnetics 39(2), 99-119 (2019)

2. Mohseni, E., Boukani, H.H., França, D.R., Viens, M.: A study of the automated eddy current detection of cracks in steel plates. J. Nondestr. Eval. 39, 6 (2020). https://doi.org/10.1007/ s10921-019-0647-9

3. Bouchala, T., Aouf, A., Abdelhadi, B., et al.: Fictitious electric conductivity modelling of eddy current evaluation of magnetic materials. Nondestr. Test. Eval. 35(1), 19-28 (2020)

4. Cao, B., Li, X., Wang, M., et al.: Analytical modelling, and simulations for high-frequency eddy current testing with planar spiral coils. Nondestr. Test. Eval. (2020). https://doi.org/10.1080/10589 759.2020.1714614

5. Mohseni, E., França, D.R., Viens, M., Xie, W.F., Xu, B.: Finite element modelling of a reflection differential split-D eddy current probe scanning surface notches. J. Nondestr. Eval. 39, 29 (2020). https://doi.org/10.1007/s10921-020-00673-6

6. Qing, Z., Xin-Jun, W.: Analytical modeling for the plate with a flat-bottom hole based on the reflection and transmission theory in pulsed eddy current testing. Acta Physica Sinica 66, 038102 (2017)

7. Bajracharya, S., Sasaki, E., Tamura, H.: Numerical study on corrosion profile estimation of a corroded steel plate using eddy current. Struct. Infrastruct. Eng. 15(9), 1151-1164 (2019)

8. Augustyniak, M., Borzyszkowski, P., Buława, M.: Towards an universal method for predicting eddy-current sensor. Characteristics in the Railway Industry. J. Nondestr. Eval. 38, 29 (2019). https:// doi.org/10.1007/s10921-019-0568-7

9. Thirunavukkarasu, S., Arjun, V., Rao, B.P.C., et al.: Development of a high-sensitive dual frequency remote field eddy current instrument for inspection of ferromagnetic steam generator tubes. IETE Tech. Rev. 36(2), 203-208 (2019)
10. Bouchala, T., Abdelhadi, B., Benoudjit, A.: Novel coupled electric field method for defect characterization in eddy current nondestructive testing systems. J. Nondestr. Eval. 33, 1-11 (2014). https://doi.org/10.1007/s10921-013-0197-5

11. Harzallah, S., Chabaat, M.: 3D-FEM computation and experimental study of eddy currents for characterization of surface cracks. Int. J. Struct. Integrity 8(5), 603-610 (2017)

12. Ibrahim, M.E., Burke, S.K.: Mutual impedance of eddy-current coils above a second-layer crack of finite length. J. Nondestr. Eval. 38, 50 (2019)

13. Zhang, Q., Wu, X.: Study on the shielding effect of claddings with transmitter-receiver sensor in pulsed eddy current testing. J. Nondestr. Eval. 38, 99 (2019). https://doi.org/10.1007/ s10921-019-0638-x

14. Yatinga, Y., Kuanhoua, G., Bowena, L., et al.: Semi-analytical method for characterization slit defects in conductingmetal by eddy current nondestructive technique. Sens. Actuators A 301, 111739 (2020)

15. Dziczkowski, L., Buchacz, A.: Modification of calibration method for conductometers. Eksploatacja i Niezawodnosc-Maint. Reliab. 4, 35-40 (2011)

16. Dziczkowski, L.: Enhancement of conductometer functions with the measurements of surface roughness. Int. J. Appl. Electromagn. Mech 41(3), 237-249 (2013)

17. Theodoulidis, T.P., Kriezis, E.E.: Eddy Current Canonical Problems (With Applications to Nondestructive Evaluation). Tech Science Press, Duluth (2006)

18. Tytko, G., Dziczkowski, L.: Fast calculation of the filamentary coil impedance using the truncated region eigenfunction expansion method. ACES J. 33(12), 1461-1466 (2018)

19. Tytko, G., Dziczkowski, L.: An analytical model of an I-cored coil located above a conductive material with a hole. Eur. Phys. J. Appl. Phys. 82, 21001 (2018)

20. Kowalczyk, P.: Complex root finding algorithm based on Delaunay triangulation. ACM Trans. Math. Softw. 41(3), 1-13 (2015)

21. Vasic, D., Ambru, D., Bilas, V.: Computation of the eigenvalues for bounded domain eddy-current models with coupled regions. IEEE Trans. Magn. 52, 7004310 (2016)

22. Kowalczyk, P.: Global complex roots and poles finding algorithm based on phase analysis for propagation and radiation problems. IEEE Trans. Antennas Propag. 66(12), 7198-7205 (2018)

23. Tytko, G., Dawidowski, Ł: Locating complex eigenvalues for analytical eddy-current models used to detect flaws. COMPEL 38, 1800-1809 (2019)

24. Theodoulidis, T., Kriezis, E.: Series expansions in eddy current nondestructive evaluation models. J. Mater. Process. Technol. 161, 343-347 (2005)

25. Tytko, G.: Fast method of calculating the air-cored coil impedance using the filamentary coil model. Prog. Electromagn. Res. M. 91, $101-109$ (2020)

Publisher's Note Springer Nature remains neutral with regard to jurisdictional claims in published maps and institutional affiliations. 